\title{
Respiratory Deposition of Fibers in the Non-inertial Regime- Development and Application of a Semi-analytical Model
}

\author{
Sofie M. Högberg ${ }^{1 *}$, Hans O. Åkerstedt ${ }^{1}$, T. Staffan Lundström ${ }^{1}$, and Jonathan B. Freund ${ }^{2}$ \\ ${ }^{1}$ Department of Applied Physics and Mechanical Engineering, Luleå University of Technology, SE-97187 Luleå, SWEDEN. \\ *corresponding author, email: sofie.hogberg@ltu.se. \\ ${ }^{2}$ Departments of Mechanical Science and Engineering and Aerospace Engineering, University of Illinois at Urbana-Champaign, \\ Urbana, IL-61801, USA.
}




\section{ABSTRACT}

A semi-analytical model describing the motion of fibrous particles ranging from nano- to micro scale was developed, and some important differences in respiratory tract transport and deposition between fibrous particles of various sizes and shapes were elucidated. The aim of this work was to gain information regarding health risks associated with inhalation exposure to small fibers such as carbon nanotubes. The model, however, is general in the sense that it can be applied to arbitrary flows and geometries at small fiber Stokes and Reynolds numbers. Deposition due to gravitational settling, Brownian motion and interception was considered, and results were presented for steady, laminar, fully developed parabolic flow in straight airways. Regarding particle size, our model shows that decrease in particle size lead to reduced efficiency of sedimentation but increased intensity of Brownian diffusion, as expected. If regarding the effects due to particle shape alone, by varying the aspect ratios and diameters of the microfibers simultaneously such that the effect of particle mass does not come into play, our model suggests that deposition both due to gravitational settling and Brownian diffusion decreases with increased fiber aspect ratio. If considering the combined effect of fiber size and shape, our results suggest that for particles with elongated shape, those with diameters in the size-range range $10-100 \mathrm{~nm}$ and lengths of several micrometers have the highest possibility to reach the vulnerable gas-exchange region in the deep lung. Note that the popular multi-walled carbon nanotubes fall into this size-range.

\section{INTRODUCTION}

Health risks associated with inhalation of toxic particles depend on the extent of exposure. Thus, knowledge of transport and deposition properties of aerosol particles in lung flows is essential. Such knowledge is also useful in the optimization of drug delivery with pharmaceutical aerosols. Hardly any particles are perfectly spherical, hence it is necessary to evaluate the implications of particle shape. Understanding the motion of fibrous particles is also of importance in other areas, including paper making, composites manufacturing, and food processing. We have learnt from history that fibers may be more harmful than spherical particles; asbestos is a notorious example of hazardous fibrous materials, and with the rapid growth of the field of nanotechnology, concern has been raised about the extended use of fibrous nanoparticles (Gogotsi, 2003; Oberdörster, Maynard, Donaldson et al., 2005; Donaldson, Aitken, Tran et al., 2006; Poland, Duffin, Kinloch et al., 2008). As a consequence, the needs for health risk assessments on nanoparticles are increased, as are the demands on knowledge about how to filter them (Åkerstedt, 
Lundström and Högberg, 2007). Carbon nanotubes (CNTs) constitute a popular class of engineered nanomaterials due to a structure that provides a combination of properties highly desirable in many products. Their high aspect ratios make the CNTs an attractive structural material, but their nanometer-scale diameter and needle-like shape have drawn comparisons with asbestos (Poland et al., 2008).

The particle mechanics of fibers is intricate because of the rotational movement involved. A common way of reducing the complexity of fiber modeling, therefore, is simply to model spheres with equivalent diameters. This approach has limited application, though, and is not truly representative for fiber flow. The drag force on a fiber depends on the instantaneous fiber orientation with respect to the flow, and hence equations for both fiber translation and rotation are required to determine fiber trajectories. In the past, Euler angles have frequently been used to compute the fiber rotation (Chen and Yu, 1991; Johnson, 1994; Tucker and Advani, 1994; Asgharian and Anjilvel, 1995a, 1995b). The group of all rotations in $\mathbb{R}^{3}$, however, cannot be represented with three parameters only. As a result, the Euler angles inevitably bring problems with singularities in the evaluation of their time rate of change, which makes them inconvenient for rigid body simulations of fibers undergoing full rotations. An alternative is to calculate the fiber rotation using quaternions (Fan and Ahmadi, 2000; Zhang, Ahmadi, Fan et al., 2001; Swaminathan, Mukundakrishnan and $\mathrm{Hu}, 2006$ ), which are algebraic constructs composed of four parameters describing evolvement of fiber orientation utilizing a unit vector for the axis of rotation and an angle of rotation around that axis. In addition to singularity-free equations of motion, the use of quaternions offers easy control of the numerical drift and may lead to reduced computational costs since only arithmetic operations are involved.

Most published work on particle transport and deposition in human lung covers spherical particles only, and available papers on non-spherical particles are limited. to particle sizes above the submicron range and/or to specific flows or geometries. Research needs, therefore, include improved understanding of nanoparticle transport and deposition in the respiratory tract, and nonspherical particle dynamics (Phalen and Hoover, 2006; Kleinstreuer and Zhang, 2010). The aims of the work presented in this paper are twofold, 1) to describe a semi-analytical model for fiber motion, valid for both nano- and microparticles in general flows and geometries, and 2) to elucidate some differences in transport and deposition properties for fibrous particles of various sizes and shapes in straight tubes representing respiratory airways. 


\section{FORCES AND TORQUES}

Two coordinate frames are defined; one global coordinate system $[x, y, z]$, fixed in space, and one local coordinate system $\left[x^{\prime}, y^{\prime}, z^{\prime}\right]$, fixed to the fiber. The fiber polar axis is along $z^{\prime}$. The rotation matrix $\mathbf{A}$ transforms from local to global coordinates; if the vector $\mathbf{x}^{\prime}$ is considered, A may be used to obtain its components in the rotated global coordinate system via $\mathbf{x}^{\prime}=\mathbf{A x}$. Note that all primed quantities refer to the fiber coordinate frame.

For the flow conditions studied, the fiber Stokes numbers are small. Thus fiber inertia may be neglected and the fiber equations of motion reduce to

$F^{h}{ }_{i}+F^{g}{ }_{i}+F^{B r}{ }_{i}=0$

$T^{h}{ }_{i^{\prime}}+T^{B r}{ }^{\prime}=0$

in which $\mathrm{F}^{\mathrm{h}}{ }_{\mathrm{i}}$ and $\mathrm{T}^{\mathrm{h}}{ }_{\mathrm{i}}$, are the hydrodynamic drag and torque, $\mathrm{F}^{\mathrm{g}}{ }_{\mathrm{i}}$ is the gravitational force, and $\mathrm{F}^{\mathrm{Br}}{ }_{i}$ and $\mathrm{T}^{\mathrm{Br}}{ }_{i}$, is the Brownian fluctuating force and torque respectively. Note that the equation for the forces is given in global coordinates, while the equation for the torques is specified in local coordinates.

\section{Hydrodynamic force and torque}

The drag force acting on a fiber depends on the current fiber orientation and is given by

$$
\boldsymbol{F}^{h}=3 \pi \mu \mathbf{A}^{T}\left[\begin{array}{ccc}
d_{\perp} & 0 & 0 \\
0 & d_{\perp} & 0 \\
0 & 0 & d_{\|}
\end{array}\right] \mathbf{A}\left[\begin{array}{c}
u-\frac{d x}{d t} \\
v-\frac{d y}{d t} \\
w-\frac{d z}{d t}
\end{array}\right]
$$

in the Stokes-flow regime, i.e. for small fiber Reynolds numbers. Here, $\mu$ is the dynamic viscosity of the fluid (air), $[u, v, w]$ are the components of the fluid velocity, and $[d x / d t, d y / d t, d z / d t]$ are the components of the fiber velocity. The parameters $d_{\|}$and $d_{\perp}$ are the Stokes diameters for a fiber oriented parallel and perpendicular to the direction of motion (Fuchs, 1964),

$$
\begin{aligned}
& \frac{d_{\|}}{d_{f}}=\frac{\frac{4}{3}\left(\beta^{2}-1\right)}{C_{\|}^{t}\left[\frac{2 \beta^{2}-1}{\sqrt{\beta^{2}-1}} \ln \left(\beta+\sqrt{\beta^{2}-1}\right)-\beta\right]}, \\
& \frac{d_{\perp}}{d_{f}}=\frac{\frac{8}{3}\left(\beta^{2}-1\right)}{C_{\perp}^{t}\left[\frac{2 \beta^{2}-3}{\sqrt{\beta^{2}-1}} \ln \left(\beta+\sqrt{\beta^{2}-1}\right)+\beta\right]},
\end{aligned}
$$


$\beta$ and $d_{f}$ being the fiber aspect ratio (ratio of length to diameter) and geometric diameter, respectively. The slip correction factors for the fluid dynamic drag, $C_{\|}{ }^{t}$ and $C_{\perp}{ }^{t}$, are included to extend the validity of the drag force to particles with diameters near the mean free path of the air molecules. No exact expressions are available for the slip correction factors on ellipsoids, therefore the approximate method by Dahneke (1973b) is applied, as briefly described in Appendix A.

The equations for the fluid dynamic torque on an ellipsoidal body moving in a viscous shear flow was derived by Jeffery (1922). For our prolate spheroid they simplify to

$$
\begin{aligned}
& T_{x^{\prime}}^{h}=\frac{1}{B_{\perp}^{r}}\left[\left(\xi^{\prime}-\omega_{x^{\prime}}\right)-\left(\frac{\beta^{2}-1}{\beta^{2}+1}\right) \dot{\varepsilon}_{y^{\prime} z^{\prime}}\right], \\
& T_{y^{\prime}}^{h}=\frac{1}{B_{\perp}^{r}}\left[\left(\eta^{\prime}-\omega_{y^{\prime}}\right)+\left(\frac{\beta^{2}-1}{\beta^{2}+1}\right) \dot{\varepsilon}_{x^{\prime} z^{\prime}}\right] .
\end{aligned}
$$

Due to the rotational symmetry of the particle, rotation about the major axis does not affect fiber transport and is therefore omitted in the analysis. In the equations above, $\omega_{i}$, are the components of the fiber angular velocity, $\dot{\varepsilon}_{i j}=\frac{1}{2}\left(\frac{\partial u_{j}}{\partial x_{i}}+\frac{\partial u_{i}}{\partial x_{j}}\right)$ are the elements of the deformation rate, and $[\xi, \eta, \zeta]^{T}=\frac{1}{2}(\nabla \times \mathbf{u})$ are the spin tensors. Both the former and the latter are received in global coordinates from the solution of the flow field, and the local equivalents are found by the transformations

$\dot{\varepsilon}_{i^{\prime} j^{\prime}}=\mathbf{A} \dot{\varepsilon}_{i j} \mathbf{A}^{T}$,

$\left[\xi^{\prime}, \eta^{\prime}, \zeta^{\prime}\right]^{T}=\mathbf{A}[\xi, \eta, \zeta]^{T}$

Further, the angular mobility is defined as

$$
B_{\perp}^{r}=\frac{3 C_{\perp}^{r}\left[\frac{2 \beta^{2}-1}{\sqrt{\beta^{2}-1}} \ln \left(\beta+\sqrt{\beta^{2}-1}\right)-\beta\right]}{2 \pi \mu d_{f}^{3}\left(\beta^{4}-1\right)} .
$$

For the slip correction factors for rotational motion we will use the approximation by Fan and Ahmadi (2000), who extended the adjusted-sphere-approximation by Dahneke to rotational motion. Due to the lack of expressions for the hydrodynamic torque on ellipsoids in the free molecule regime, the evaluation is made for cylinders. For $\beta » 1$ the rotational slip correction factors for cylinders may serve as approximations for ellipsoids. The adjusted radius for the rotational motion is given in Appendix A. 


\section{Gravitation}

The particle density is much greater than the density of the surrounding air, and thus buoyancy is neglected. Gravity is defined in the positive $z$-direction with magnitude

$F_{z}^{g}=\pi \rho_{f} g d_{f}^{3} \beta / 6$

where $\rho_{f}$ is the fiber density and $g$ is the gravitational acceleration.

\section{Brownian motion}

The components of the Brownian force and torque are modeled as independent zero-mean Gaussian white-noise processes (Fan and Ahmadi, 2000), and for a prolate spheroid they can be written in the form

$$
\begin{aligned}
& F^{B r}{ }_{i}(t)=\chi_{i^{\prime}}{ }^{t} \sqrt{\frac{\pi S_{i^{t}}}{\delta t}}, \\
& T^{B r}(t)=\chi_{i^{\prime}}{ }^{r} \sqrt{\frac{\pi S_{i^{\prime}}{ }^{r}}{\delta t}},
\end{aligned}
$$

where $\chi_{i}^{t}$, and $\chi_{i}^{r}$, are standard normally distributed random numbers, and $\delta t$ is a Brownian time increment. The spectral intensities appearing in Eqs. (11)-(12) are given by

$$
\begin{aligned}
& S_{i^{\prime}}{ }^{t}=\frac{2 \sigma T}{\pi} \frac{1}{B^{t}{ }^{\prime}}, \\
& S_{\perp}{ }^{r}=\frac{2 \sigma T}{\pi} \frac{1}{B^{r}{ }_{\perp}},
\end{aligned}
$$

in which $\sigma$ is the Boltzmann constant and $T$ is the absolute temperature. The angular mobility was given in Eq. (9), and the translational mobility is defined as

$$
B_{i^{\prime}}{ }^{t}=1 / 3 \pi \mu d_{i^{\prime}} .
$$

\section{ROTATION MATRIX AND EQUATIONS FOR RIGID-BODY ROTATION}

The equations for fiber movement include both locally and globally defined variables, and to enable transformation between the two coordinates frames we need to derive a rotation matrix. Any rotation about a fixed point can be expressed in the form (Goldstein, 1980; Rapaport, 1997)

$\mathbf{r}=\mathbf{r}^{\prime} \cos \alpha+\left(\mathbf{c} \cdot \mathbf{r}^{\prime}\right) \mathbf{c}(1-\cos \alpha)+\left(\mathbf{c} \times \mathbf{r}^{\prime}\right) \sin \alpha$, 
where $\mathbf{c}$ is a unit vector along the axis of rotation and $\alpha$ is the rotation angle. Quaternions are based upon sets of four parameters, known as Euler parameters, and by defining these as $q_{4}=\cos (\alpha / 2)$ and $q_{m}=c_{m} \sin (\alpha / 2)$ for $m=1,2,3$, Eq. (16) takes the form

$$
\mathbf{r}=\left(2 q_{4}^{2}-1\right) \mathbf{r}^{\prime}+2\left(\mathbf{q} \cdot \mathbf{r}^{\prime}\right) \mathbf{q}+2 q_{4}\left(\mathbf{q} \times \mathbf{r}^{\prime}\right) .
$$

Further, Eq. (17) can be expressed as $\mathbf{r}=\mathbf{A}^{\mathrm{T}} \mathbf{r}$, leading directly to the orthogonal rotation matrix

$$
\mathbf{A}=2\left[\begin{array}{ccc}
q_{1}^{2}+q_{4}^{2}-\frac{1}{2} & q_{1} q_{2}+q_{3} q_{4} & q_{1} q_{3}-q_{2} q_{4} \\
q_{1} q_{2}-q_{3} q_{4} & q_{2}^{2}+q_{4}^{2}-\frac{1}{2} & q_{2} q_{3}+q_{1} q_{4} \\
q_{1} q_{3}+q_{2} q_{4} & q_{2} q_{3}-q_{1} q_{4} & q_{3}^{2}+q_{4}^{2}-\frac{1}{2}
\end{array}\right],
$$

which is algebraically equivalent to the more frequently encountered rotation matrix composed of Euler angles, here received upon application of three successive rotations $(\phi, \theta, \psi)$ using the $x$-convention (Goldstein, 1980),

$\mathbf{A}=\left[\begin{array}{ccc}c \psi c \phi-c \theta s \phi s \psi & c \psi s \phi+c \theta c \phi s \psi & s \theta s \psi \\ -s \psi c \phi-c \theta s \phi c \psi & c \theta c \phi c \psi-s \psi s \phi & s \theta c \psi \\ s \theta s \phi & -s \theta c \phi & c \theta\end{array}\right]$

where $c \phi=\cos \phi, s \phi=\sin \phi$. etc. The Euler parameters are related to the Euler angles by (Goldstein, 1980; Rapaport, 1997)

$q_{1}=\sin \frac{\theta}{2} \cos \frac{\phi-\psi}{2}$,

$q_{2}=\sin \frac{\theta}{2} \sin \frac{\phi-\psi}{2}$

$q_{3}=\cos \frac{\theta}{2} \sin \frac{\phi+\psi}{2}$,

$q_{4}=\cos \frac{\theta}{2} \cos \frac{\phi+\psi}{2}$

and are also subjected to the identity

$\sum_{n} q_{n}^{2}=1$

which can be used for normalization in every time-step, thereby offering control of the numerical drift.

We need to find expressions for the rate of change of the quaternions in order to update the rotation matrix in every time-step. This can be achieved by first defining a quaternion as the complex sum of a scalar and a vector, $\tilde{\mathbf{q}}=q_{4}+\mathbf{i q}$, 
where $\mathbf{q}=\left[q_{1}, q_{2}, q_{3}\right]^{\mathrm{T}}$. Then, after some calculations and simplifications using quaternion algebra (Goldstein, 1980; Rapaport, 1997) we arrive at

$$
\left[\begin{array}{c}
\dot{q}_{1} \\
\dot{q}_{2} \\
\dot{q}_{3} \\
\dot{q}_{4}
\end{array}\right]=\frac{1}{2}\left[\begin{array}{cccc}
q_{4} & -q_{3} & q_{2} & q_{1} \\
q_{3} & q_{4} & -q_{1} & q_{2} \\
-q_{2} & q_{1} & q_{4} & q_{3} \\
-q_{1} & -q_{2} & -q_{3} & q_{4}
\end{array}\right]\left[\begin{array}{c}
\omega_{x^{\prime}} \\
\omega_{y^{\prime}} \\
\omega_{z^{\prime}} \\
0
\end{array}\right]
$$

showing that the quaternions can be updated simply from knowledge about the fiber angular velocity.

\section{NONDIMENSIONAL EQUATIONS OF MOTION}

Nondimensional parameters are defined as $u_{i}{ }^{*}=u_{i} / U, x_{i}{ }^{*}=x_{i} / R$, and $t^{*}=t U / R$, where $R$ and $U$ is respectively a characteristic length and velocity of the fluid flow. For flow in the respiratory airways, $R$ is the radius of the current airway and $U$ is the average velocity in the same.

Solution of Eq. (1) for the fiber velocity on dimensionless form yields

$$
\left[\begin{array}{l}
\frac{d x^{*}}{d t^{*}} \\
\frac{d y^{*}}{d t^{*}} \\
\frac{d z^{*}}{d t^{*}}
\end{array}\right]=\left[\begin{array}{c}
u^{*} \\
v^{*} \\
w^{*}
\end{array}\right]+\mathbf{A}^{T}\left[\begin{array}{ccc}
\frac{d_{t}}{d_{\perp}} & 0 & 0 \\
0 & \frac{d_{t}}{d_{\perp}} & 0 \\
0 & 0 & \frac{d_{f}}{d_{\| \prime}}
\end{array}\right] \mathbf{A}\left[\begin{array}{l}
0 \\
0 \\
k
\end{array}\right]+\mathbf{A}^{T}\left[\begin{array}{l}
v_{B r, x^{*}}\left(t^{*}\right) \\
v_{B r, y^{*}}\left(t^{*}\right) \\
v_{B r, z^{*}}\left(t^{*}\right)
\end{array}\right] .
$$

The second term on the right-hand side of this system of equations represents the fiber settling velocity, with the dimensionless sedimentation parameter $k$ given by

$$
k=\frac{\rho_{f} g d_{f}^{2} \beta}{18 \mu U}
$$

where $\rho_{f}$ is the fiber density. Holding $k$ constant has the same effect on the sedimentation velocity as keeping the mass constant, and larger mass means larger $k$. The third term represents the Brownian diffusion velocity,

$v_{B r, i^{\prime}}{ }^{*}\left(t^{*}\right)=\chi_{i^{*}}{ }^{t} \sqrt{\frac{2 D_{i^{\prime}}{ }^{*}}{\delta t^{*}} \frac{1}{U R}}$,

in which

$$
D_{i^{\prime}}{ }^{t}=\sigma T B_{i^{\prime}}{ }^{t}
$$

is the translational diffusion coefficient. The Brownian diffusion velocity is proportional to $d_{f}^{-1 / 2}$ approximately, and thus it is rapidly decreasing with increased particle size. If the rotation matrices in Eq. (27) are developed, we finally get 


$$
\begin{aligned}
\frac{d x^{*}}{d t^{*}} & =u^{*}+4 k\left[\left(\frac{d_{f}}{d_{\|}}-\frac{d_{f}}{d_{\perp}}\right)\left(q_{1} q_{3}+q_{2} q_{4}\right)\left(\frac{1}{2}-q_{1}^{2}-q_{2}^{2}\right)\right] \\
& +2\left[v^{*}{ }_{B r, x^{\prime}}\left(q_{1}^{2}+q_{4}^{2}-\frac{1}{2}\right)+v_{B r, y^{\prime}}^{*}\left(q_{1} q_{2}-q_{3} q_{4}\right)+v_{B r, z^{\prime}}^{*}\left(q_{1} q_{3}+q_{2} q_{4}\right)\right] \\
\frac{d y^{*}}{d t^{*}} & =v^{*}-4 k\left[\left(\frac{d_{f}}{d_{\|}}-\frac{d_{f}}{d_{\perp}}\right)\left(q_{1} q_{4}-q_{2} q_{3}\right)\left(\frac{1}{2}-q_{1}^{2}-q_{2}^{2}\right)\right] \\
& +2\left[v_{B r, x^{\prime}}^{*}\left(q_{1} q_{2}+q_{3} q_{4}\right)+v_{B r, y^{\prime}}^{*}\left(q_{2}^{2}+q_{4}^{2}-\frac{1}{2}\right)+v_{B r, z^{\prime}}^{*}\left(q_{2} q_{3}-q_{1} q_{4}\right)\right] \\
\frac{d z^{*}}{d t^{*}} & =w^{*}+4 k\left[\frac{d_{f}}{d_{\|}}\left(\frac{1}{2}-q_{1}^{2}-q_{2}^{2}\right)^{2}+\frac{d_{f}}{d_{\perp}}\left(q_{1}^{2}+q_{2}^{2}\right)\left(q_{3}^{2}+q_{4}^{2}\right)\right] \\
& +2\left[v_{B r, x^{\prime}}^{*}\left(q_{1} q_{3}-q_{2} q_{4}\right)+v_{B r, y^{\prime}}^{*}\left(q_{2} q_{3}+q_{1} q_{4}\right)+v_{B r, z^{\prime}}^{*}\left(\frac{1}{2}-q_{1}^{2}-q_{2}^{2}\right)\right] .
\end{aligned}
$$

Substitution of Eqs. (6)-(7) and (12) for the hydrodynamic and Brownian torques into Eq. (2) for the fiber rotation and solving for the angular velocity yields

$$
\begin{aligned}
& \omega_{x^{\prime}}{ }^{*}=\xi^{*^{*}}-\frac{\beta^{2}-1}{\beta^{2}+1} \dot{\varepsilon}_{y^{\prime} z^{\prime}}{ }^{*}+\chi^{r}{ }_{x^{\prime}} \sqrt{\frac{2 D_{\perp}^{r}}{\delta t^{*}} \frac{R}{U}}, \\
& \omega_{y^{\prime}}{ }^{*}=\eta^{\prime^{*}}+\frac{\beta^{2}-1}{\beta^{2}+1} \dot{\varepsilon}_{x^{\prime} z^{\prime}}{ }^{*}+\chi^{r}{ }_{y^{\prime}} \sqrt{\frac{2 D_{\perp}^{r}}{\delta t^{*}} \frac{R}{U}},
\end{aligned}
$$

in which

$$
D_{\perp}^{r}=\sigma T B_{\perp}^{r}
$$

is the rotational diffusion coefficient.

\section{SOLUTION PROCEDURE}

If considering submicron fibers, the equations for fiber movement contain nondeterministic terms because of the randomness in the Brownian motion. For this reason, the advancement of the fiber position with time requires solution of a set of stochastic differential equations (SDEs). The Euler-Maruyama (EM) method is a generalization of the ordinary Euler method and represents the simplest strong Taylor approximation (Kloeden and Platen, 1999; Higham, 2001). In this work, Eqs. (26) and (27) for the fiber rotation and translation are solved with MATLAB (Mathworks Inc.) using the Euler-Maruyama scheme, and if omitting the Brownian terms in the equations of motion, a solver for ordinary differential equations, like Runge-Kutta $(4,5)$, is shown to provide the same results. The numerical time-step is set to $0.1 \mathrm{~ms}$, reduction of the time-step did not affect the fiber deposition efficiency. The 
initial position of the fiber center of mass is specified with dimensionless Cartesian coordinates, and initial fiber orientation is provided with Euler angles.

\section{Deposition criterion}

The deposition criterion for a spherical particle is a function of the position of center of mass only, whereas a fiber, due to its elongated shape, may be deposited even though its center of mass is relatively far from a wall. Interceptive deposition increases in importance as fiber lengths approach the size of the bounding geometry, the airway diameter in this context. Fibers are oriented along $[0,0,1]$ in local coordinates in the current configuration of the coordinate system, whereby the fiber orientation in global coordinates is given by

$\mathbf{r}_{\text {rot }}=A^{T}[0,0,1]^{T}$,

and upon normalization, the components of the orientation vector are given by

$$
\left[\begin{array}{l}
x_{\text {rot }} \\
y_{\text {rot }} \\
z_{\text {rot }}
\end{array}\right]=\frac{2}{\left|r_{\text {rot }}\right|}\left[\begin{array}{c}
q_{1} q_{3}+q_{2} q_{4} \\
q_{2} q_{3}-q_{1} q_{4} \\
q_{3}^{2}+q_{4}^{2}-\frac{1}{2}
\end{array}\right]
$$

The fiber length is defined as $L_{f}=\beta d_{f}$, whereby the fiber endpoints are localized at

$\mathbf{x}_{e p}=\mathbf{x}_{c} \pm \frac{1}{2} L_{f} \mathbf{x}_{r o t}$,

with $\boldsymbol{x}_{c}$ being the position of the fiber center of mass. A fiber is considered deposited as soon as its tip touches a wall, and no resuspension occurs. To exemplify, the deposition criterion for a fiber located in a horizontal airway with radius $R$ is $x_{e p}{ }^{2}+z_{e p}{ }^{2} \geq R^{2}$ if the airway axis is in the $y$-direction.

\section{Numerical setup}

Results are presented for steady, laminar, fully developed parabolic flow in straight horizontal and inclined airways with dimensions according to Weibel's model A (Weibel, 1963). We consider inhalation at tracheal flow rates of 431 and $833 \mathrm{~cm}^{3} / \mathrm{s}$, corresponding to tidal volumes of 750 and $1450 \mathrm{ml}$, respectively, and an inhalation time of $1.74 \mathrm{~s}$, as proposed in the ICRP model (1966). We consider unit density fibers, and air at $310 \mathrm{~K}$. To obtain results that are independent of fiber initial conditions, deposition predictions are based upon simulations of 100000 particles. Further increase in particle number was shown not to affect the results. Fibers were released one at the time, with random initial position and orientation. The deposition efficiency is defined as the ratio of the number of particles that are deposited in an airway of a specified lung generation to the number of particles that are released into that 
airway. If not stated otherwise, simulations were run for 10 times the mean residence time in an airway of given generation; the fraction of particles that were either captured or traversed the airway in that time summed to nearly $100 \%$.

\section{RESULTS AND DISCUSSION}

This section is divided into separate parts for clarity. First, results for fiber behavior as obtained simply by examining the fiber equations of motion are presented. Then we illustrate fiber transport properties, and their dependence on flow and particle characteristics, which also aids in the understanding of the deposition properties. Finally, we present results from simulations on fiber deposition, including comparison with other models, and elucidate some important differences in deposition properties of micro- and nanofibers of different shapes.

Many of the figures included in the results are intended to illustrate specific features of fiber transport and deposition, and not to overload graphs with information, results are often shown for a single lung generation, for one flow rate etc. The dependence of these parameters are presented separately, however. So that we do not need to provide the same information over and over, if not stated otherwise, the prescribed conditions are: light activity breathing, horizontally oriented airway, random fiber initial position and orientation, and airway generation 16 (representing the terminal bronchiole). Please note that in the figures illustrating fiber transport, fiber lengths are set to fixed values for illustrative purposes, and deposition by interception is not shown.

\section{Analysis of the fiber equations of motion}

If considering fibers of different sizes and shapes, characterized by fiber diameter and aspect ratio, it is apparent from Eqs. (27)-(30) that the efficiencies of gravitational settling and Brownian diffusion are functions of the sedimentation parameter $k$ and the translational diffusion coefficient $D_{i}{ }^{,}{ }^{t}$, respectively. Regarding the influence of particle size, it is expected that the efficiency of sedimentation increases with particle diameter, whereas the intensity of Brownian diffusion decreases with the same. Thus sedimentation is an important deposition mechanism for larger particles, here referred to as microparticles, whereas the gravitational motion of nanoparticles is negligible due to the small particle masses encountered, and the deviations from the fluid streamlines are solely due to Brownian motion. Regarding particle shape, the descending speed of a microfiber with a certain sedimentation parameter $k$ is a function of the reciprocal Stokes' diameters, cf. Eq. (33). The Stokes' diameters are positively correlated with the fiber aspect ratio, thus the descending speed of a microfiber decreases with increased $\beta$, independent of fiber 
orientation, indicating reduced probability of deposition due to gravitational settling for increased fiber aspect ratio, provided the diameters are varied simultaneously such that the effect of particle mass does not come into play. It can also be noted from Eqs. (31)-(33) for the fiber translation that the motion of non-spherical particles due to the gravitational force may have non-zero components in all three coordinate directions, which may result in a small drift motion. As to the nanofibers, the translational diffusion coefficients decrease with increased $\beta$, cf. Fig. 1 . Reduced intensity of the Brownian diffusion in turn leads to smaller fluctuations, see Eq. (29), and thereby to less likelihood of hitting an airway wall

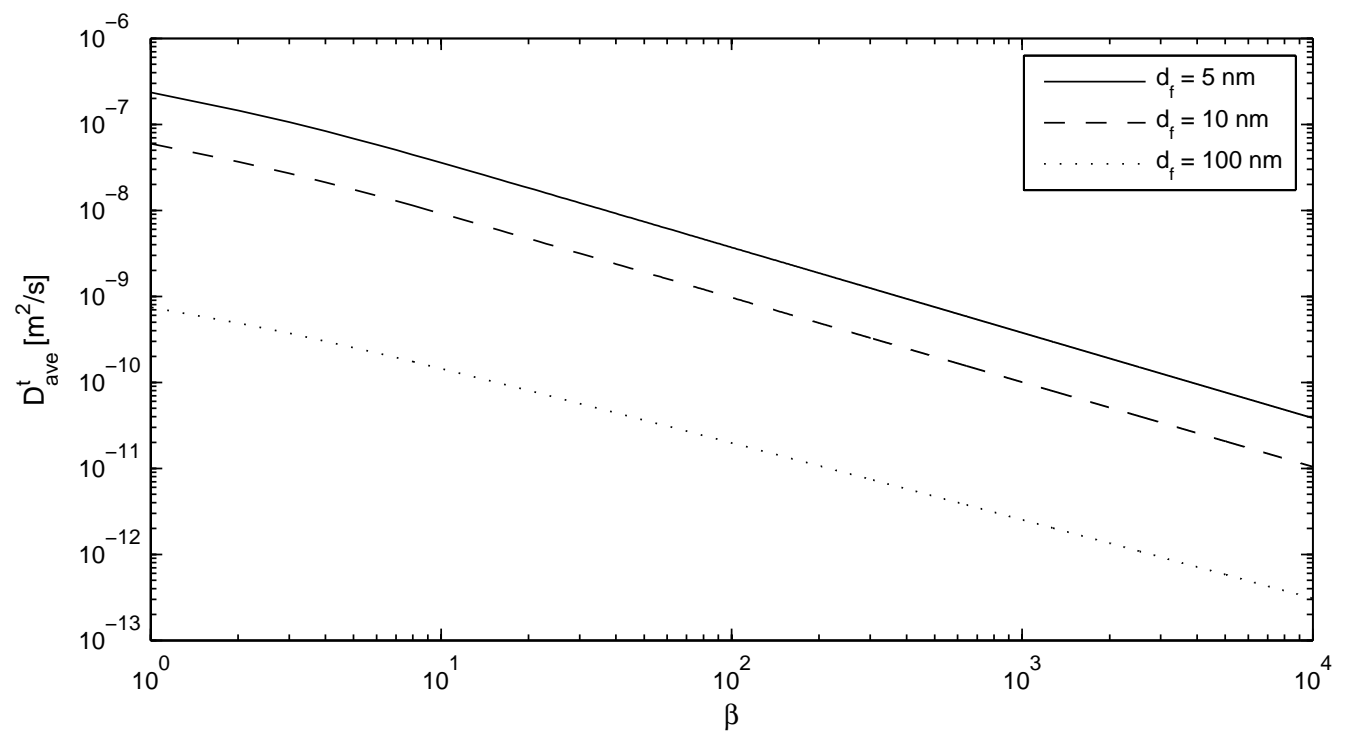

Figure 1. The effect of the fiber aspect ratio on the translational diffusion coefficient when averaged over all directions. The characteristic length and velocity are representative for the terminal bronchiole in human lung (Weibel's G16).

So far we have only discussed the fiber translational motion and its dependence of particle characteristics, but the instantaneous fiber velocity is also dependent on fiber orientation, see Eqs. (31)-(33). The rotational motion of the fiber in turn is both dependent on characteristics of the flow and of the particle, since it has contributions from fluid shear as well as Brownian rotation, see Eqs. (34)-(35). In a parabolic flow, a fiber rotates due to the presence of fluid dynamic torque and tends to align with the flow. The stochastic rotation, however, tends to counteract any orientation preferences due to its random nature. The relative importance of the two rotational mechanisms depend on the degree of shear and on the intensity of the stochastic rotations, and can be estimated with the rotational Peclet number,

$P e^{r}=G / D_{\perp}^{r}$, 
in which the maximum magnitude of the velocity gradient, $G$, is $4 U / R$ for parabolic flow. For the transport of microfibers at conditions relevant for human airways, $P e^{r} \gg 1$ due to the small rotational diffusion coefficients encountered, and the rotational motion is due to fluid shear only. For nanofibers on the other hand, the size of $P e^{r}$ may vary significantly due to its sensitivity to particle characteristics, and $P e^{r}$ then provides an estimate of the capacity of the flow to control the fiber orientation. As shown in Fig. 2 for G16, this capacity increases dramatically with both $d_{f}$ and $\beta$; please notice, however, that $\beta$ needs to be rather large to achieve $P e^{r}>1$ for the nanofibers, and if considering more proximal airways even larger $\beta$ are required due to larger velocity gradients.

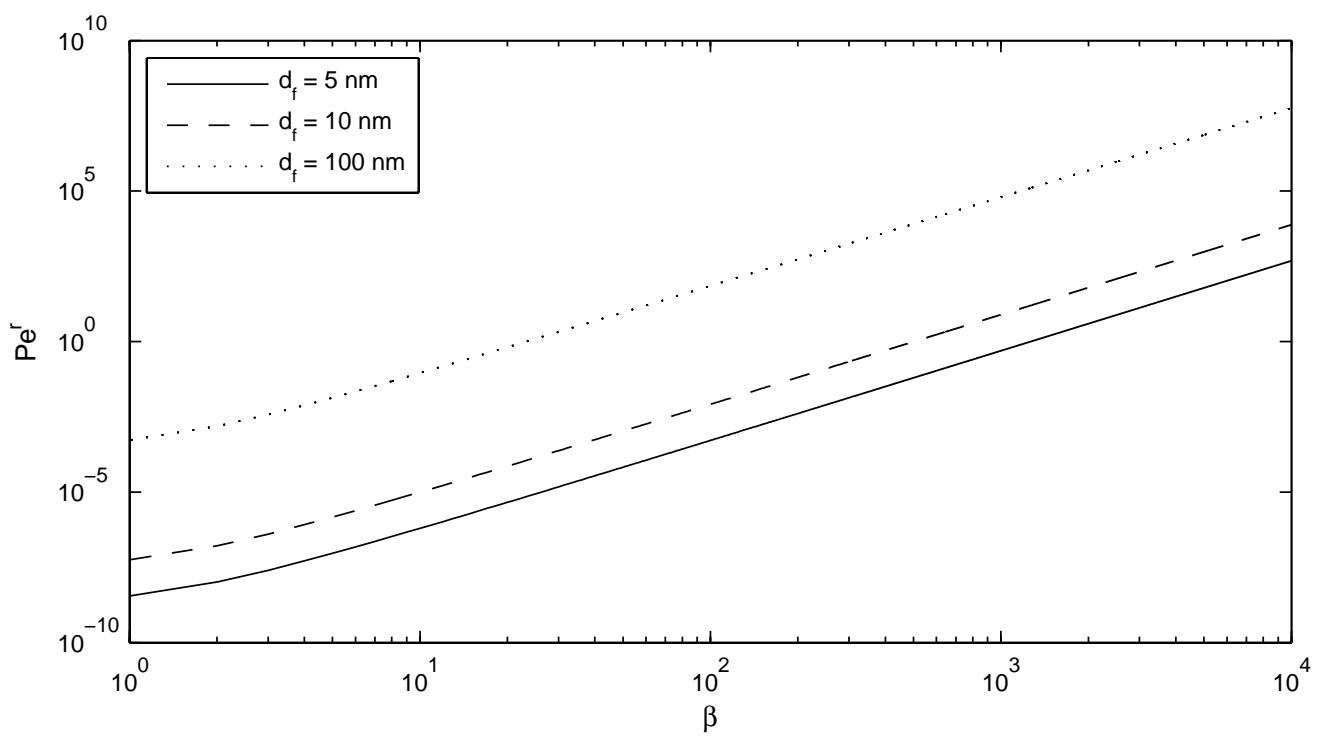

Figure 2. The influence of the fiber aspect ratio on the rotational Peclet number for nanofibers with different diameters.

\section{Simulations on fiber transport}

The descending speed of a microfiber decreases with increased $\beta$ because the fiber aligns with the flow, see Fig. 3 , which is in agreement with the discussion above. For the fibers and flow field considered here, the rotational Peclet number is of order $10^{5}-10^{6}$, i.e., the rotational motion is due to fluid shear only. The motion of a prolate ellipsoid of revolution in a shear flow is periodic (Jeffery, 1922) and the fiber rotates in so called Jeffery orbits. The orbital period for such a particle in a simple shear flow with constant shear rate $\gamma$ is

$T_{2 \pi}=\frac{2 \pi}{\gamma} \frac{\beta^{2}+1}{\beta}$ 
stating that a fiber with larger $\beta$ has longer rotation time, and at a local level Eq. (41) applies to our flow field as well. The rotational velocity is highest when the fiber is aligned perpendicular to the flow direction, and decreases as the fiber becomes parallel with the flow.

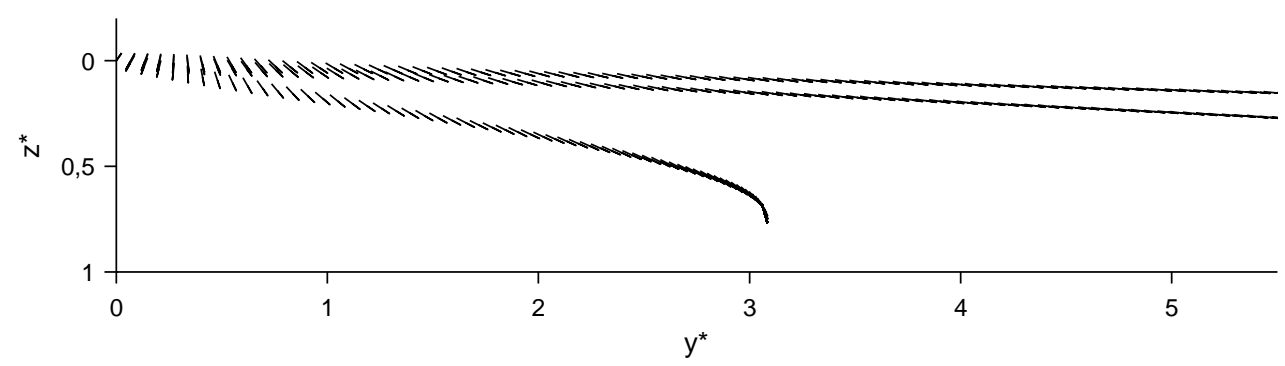

Figure 3. Trajectories of microfibers $(k=0.5$ ) with $\beta=100$ (top), $\beta=50$ (middle), and $\beta=10$ (bottom) in a horizontal airway of G16. Initial condition $\mathrm{x}^{*}=0.75, \mathrm{z}^{*}=0, \phi=-\pi / 4, \theta=\pi / 4, \psi=0$.

The intensity of the Brownian motion can be estimated with the root-mean-square Brownian displacement and rotation. The 1-D rms net Brownian displacement along the $x^{\prime}$ - axis during time $t$, for example, is

$$
x^{\prime}{ }_{r m s}=\sqrt{2 D_{\perp}{ }^{t} t}
$$

and the rms rotated angle about the same axis is

$\theta_{x^{\prime}, r m s}=\sqrt{2 D_{\perp}^{r} t}$

Both the translational and rotational diffusion coefficients decrease with increased $d_{f}$ and $\beta$, consequently the size of the Brownian fluctuations and the intensity of the stochastic rotations also decrease, as shown in Figs. 4 and 5.
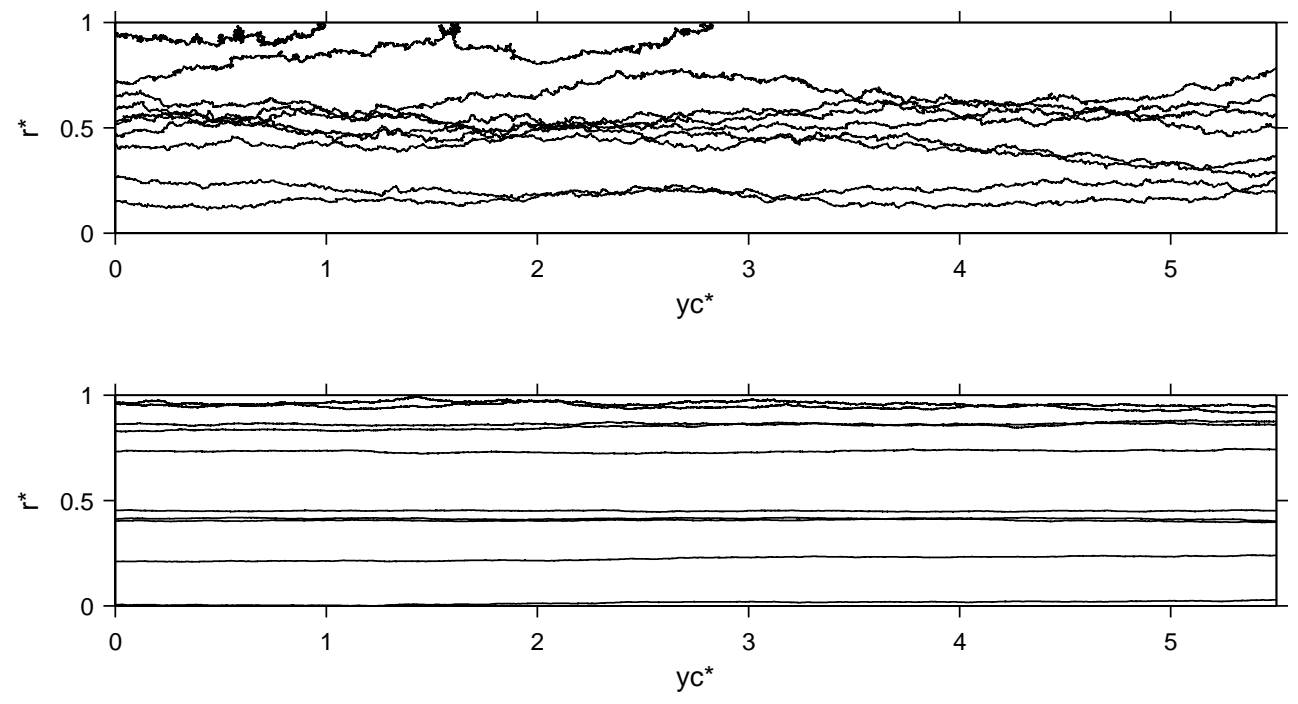


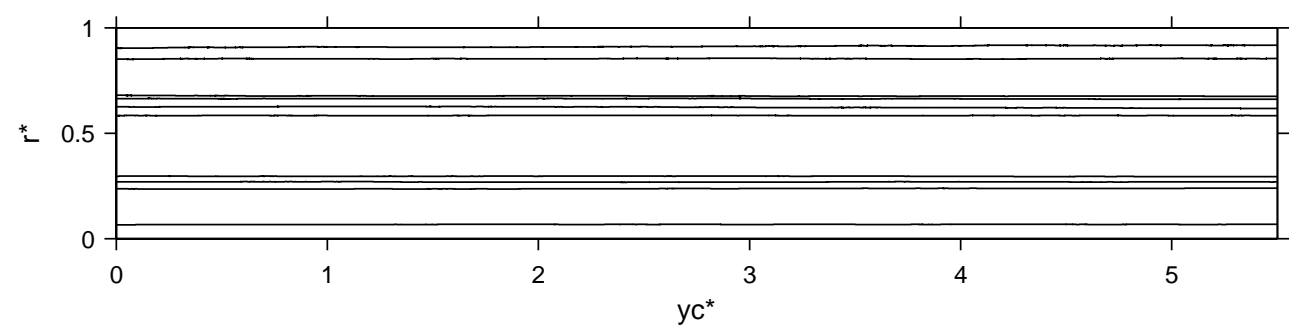

Figure 4. Radial position of center of mass for ten individual nanofibers $\left(d_{f}=10 \mathrm{~nm}\right)$ with aspect ratios $\beta=5$ (top), $\beta=$ 500 (middle), and $\beta=10000$ (bottom). $y c^{*}=$ non-dimensional coordinate for the position of the fiber center of mass along the airway.

At small $P e^{r}$, the rotational motion is mainly Brownian, which leads to a random distribution of the fiber orientation, while at large $P e^{r}$ the rotation is deterministic since it is dominated by the fluid dynamic torque. The dependence of the Peclet number on the rotational behavior is illustrated in Fig. 5, where $P e^{r}$ is of the order $10^{-6}, 1$, and $10^{3}$ for $\beta=$ 5, $\beta=500$ and $\beta=10000$, respectively. Multi-walled carbon nanotubes (MWCNTs) are usually around $10 \mathrm{~nm}$ in diameter and several micrometers in length, implying a $\beta$ between 200 and 1000. Fan and Advani (2005) observed that shear forces in a non-saturated vinyl ester align $1 \mu \mathrm{m}$ long MWCNTs in the flow direction, and shear flow induced CNT orientation is reported by others as well. Our results, however, show that only very long nanofibers are aligned with the flow, cf. Fig. 5. This emphasizes that the rotational behavior is not determined by fiber characteristics alone. Recall that the rotational Peclet number is also a function of fluid properties, see Eq.(40) along with Eqs. (36) and (9); in this example, the sensitivity of $P e^{r}$ to the dynamic viscosity of the fluid is highlighted (the viscosity of vinyl-ester being several magnitudes larger than that of air).

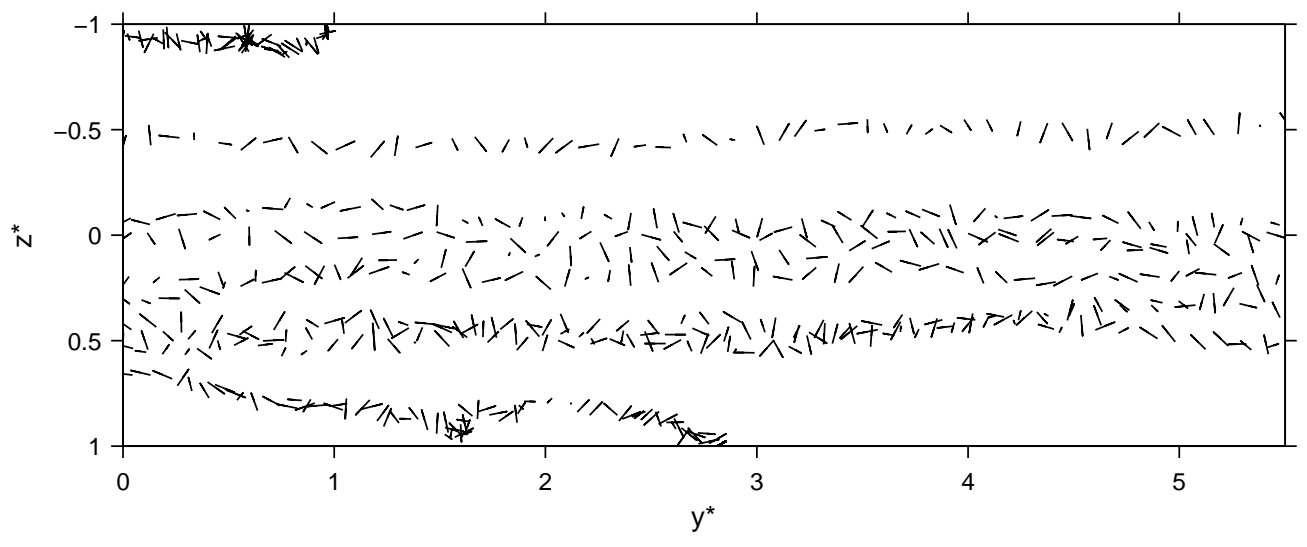



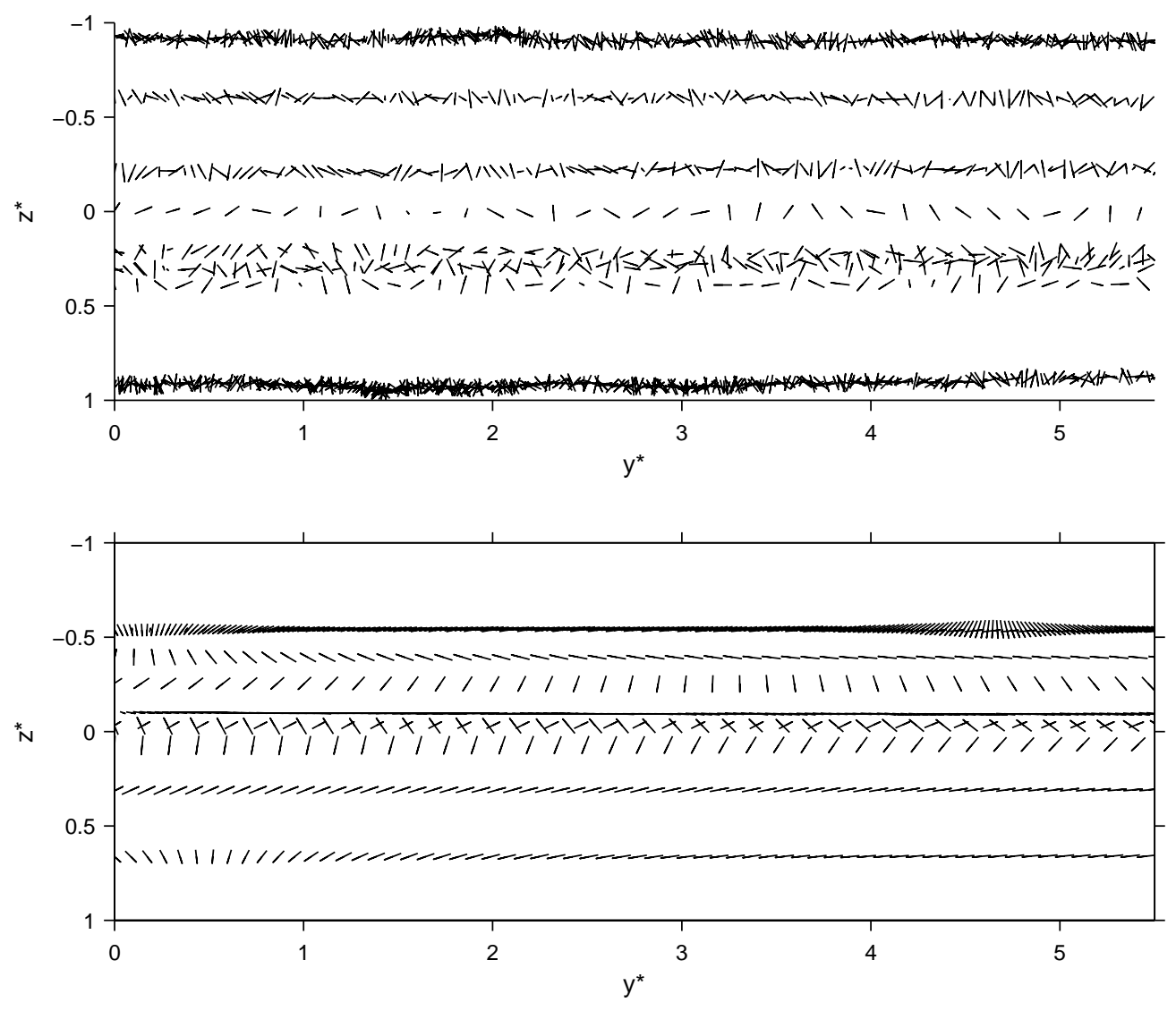

Figure 5. Particle trajectories in the xy-plane showing the rotational behavior of the fibers in Fig. 4.

\section{Simulations on fiber deposition}

The problem of deposition of spherical particles for laminar flow in a circular, horizontal tube, neglecting the inertial and Brownian motion of particles, was solved by Pich (1972) using an analytical calculation method based on the analysis of limiting trajectories of particles. For Poiseuille flow, the fraction of particles depositing in the tube was given on the form

$P_{s}=\frac{2}{\pi}\left[2 \kappa \sqrt{1-\kappa^{2 / 3}}-\kappa^{1 / 3} \sqrt{1-\kappa^{2 / 3}}+\sin ^{-1} \kappa^{1 / 3}\right]$,

with $\kappa$ being a dimensionless parameter of deposition defined as

$\kappa=\frac{3 L v_{\text {settl }}}{8 U R}$,

where $v_{\text {sett }}$ is the particle settling velocity and $L$ is the length of the tube. For non-spherical particles the settling velocity is dependent on particle orientation, and for a prolate spheroid it is given by 
$v_{\text {settle,\| }}=\frac{\rho_{f} g \beta d_{f}^{2}}{18 \mu\left(d_{\|} / d_{f}\right)}$,

$v_{\text {settle, }}=\frac{\rho_{f} g \beta d_{f}^{2}}{18 \mu\left(d_{\perp} / d_{f}\right)}$

for a fiber directed parallel and perpendicular to the direction of motion respectively. Simulations for various $\beta$ and $k$ were performed for an airway with aspect ratio $L / R=3$, and results were compared with the formula of Pich. The connection between $k$ and the settling velocity is obvious from Eq. (28), and the desired value of $k$ for a certain $\beta$ was obtained by adjusting the fiber diameter accordingly. As can be seen in Fig. 6, the simple expression by Pich give relatively good results for the case of Poiseuille flow in a horizontal pipe. For the settling velocity in Eq. (45), we have used the mean of Eqs. (46)-(47) when averaged over all directions, which by definition is equal to using the mean aerodynamic diameter in the formula for the spherical particle since we consider fibers with specific gravity of one. Pich is not the only one that has proposed a simple formula for the estimation of deposition due to sedimentation, Yeh, Schum and Duggan (1979), for instance, come up with the expression

$P_{s, 2}=1-\exp \left[\frac{-g \rho_{p} L d_{p}^{2} C_{p}}{9 \pi \mu R U}\right]$

If we adjust this expression to fibers using the aerodynamic diameter, $d_{a e}$, defined as

$$
d_{a e}=d_{f} \sqrt{\frac{1}{C_{a e}} \frac{\rho_{f}}{\rho_{o}} \beta\left[\frac{1}{3} \frac{d_{f}}{d_{\|}}+\frac{2}{3} \frac{d_{f}}{d_{\perp}}\right]},
$$

if averaged over all directions, we get results close to those received using the formula of Pich for random fiber orientation. The formula by Yeh et al. constitute one of two options offered for the prediction of fiber deposition due to sedimentation in (Sturm and Hofmann, 2006), for instance, who developed an easy-to-use computer program predicting fiber deposition in the respiratory tract. In the program the user can choose between parallel, perpendicular, and random fiber orientation on a regional level, whereby the corresponding aerodynamic diameter is used in the calculations. A limitation of these kind of deposition models, however, is that the user has to state the fiber orientation initially, and that a fiber is assumed to keep its initial orientation state over several lung generations. 


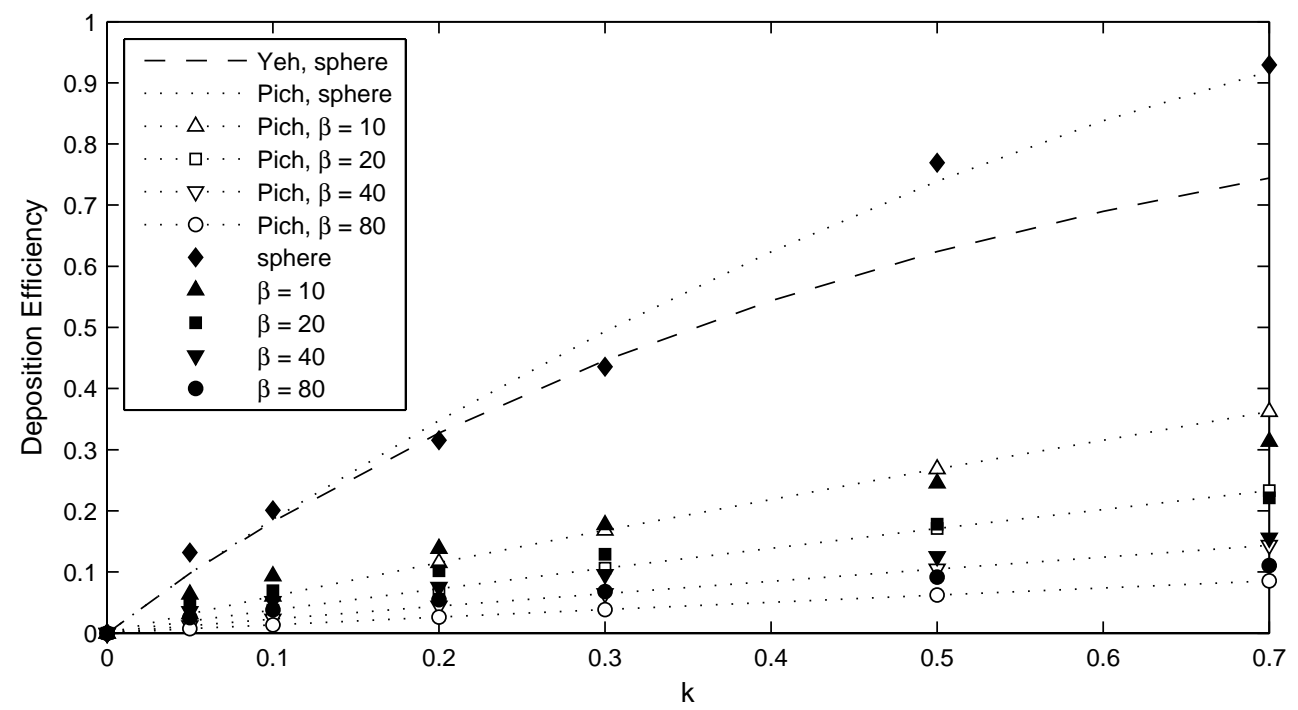

Figure 6. Deposition due to sedimentation in a horizontal pipe. Results are compared with the expression by Pich (1972), for a spherical particle and adjusted for fibers, and by Yeh et al. (1979) for a spherical particle.

The results for fiber sedimentation was also compared with the results of Asgharian \& Anjilvel (1995a), who predicted fiber deposition due to sedimentation and interception in straight, horizontal and inclined circular tubes by solving the equations of fiber translation and rotation numerically. Results were presented for the dimensionless sedimentation parameter $\tau_{1}$, which is equal to our parameter $k$ times the Cunningham correction factor. The correction factor is dependent on fiber orientation, and in the comparison presented here, the mean of the correction factor when averaged over all directions is used. Overall, the results of our study and those presented in the paper of comparison were in good agreement, see Fig. 7, and differences between the results may be partly attributed to the correction factor, which was not specified in the work by Asgharian \& Anjilvel. Further, to resemble the conditions of these authors, a fixed fiber length of $5 \mu \mathrm{m}$ with varying aspect ratio was used in the calculations, and the airway length to radius ratio was set to 3 . 


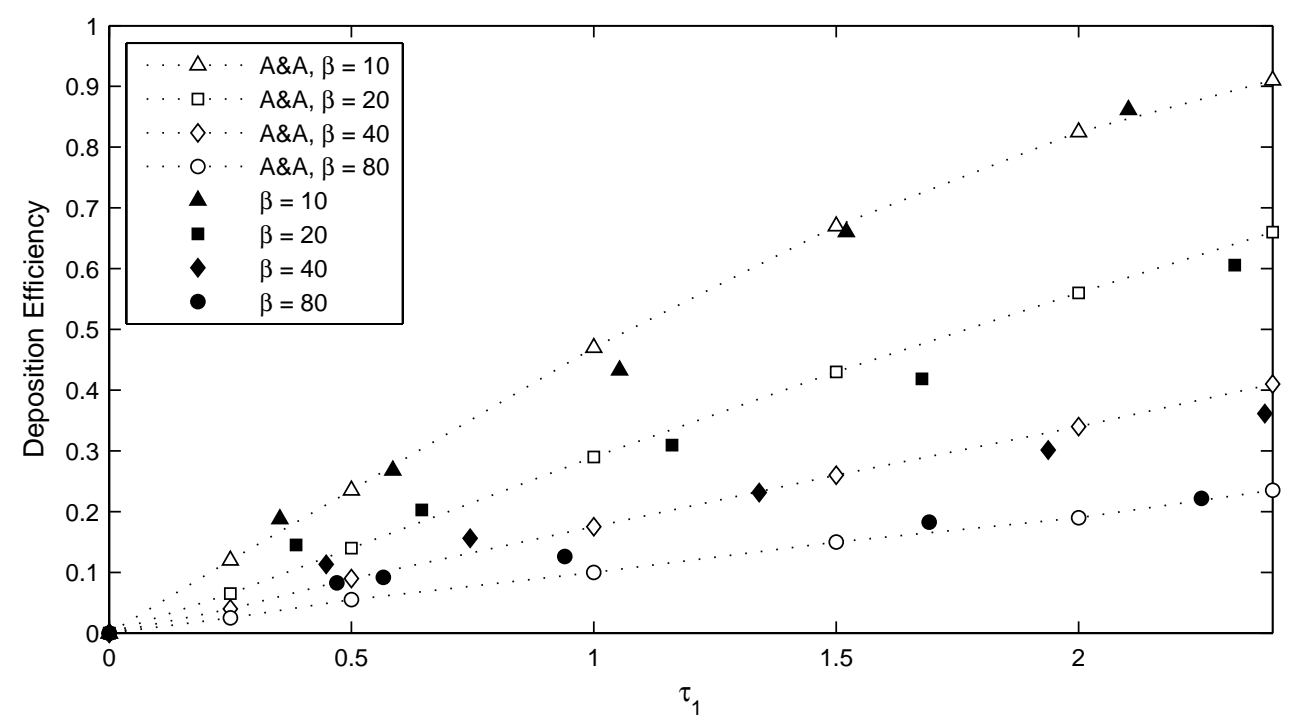

Figure 7. Deposition due to sedimentation in a horizontal tube. Comparison with results of Asgharian \& Anjilvel (1995a).

The deposition due to diffusion in the respiratory tract for a given flow field can be estimated either by solving the particle equations of motion within this flow field, as in our model, or by solving a convection-diffusion equation for the aerosol concentration. Ingham (1975) among others used the latter approach and obtained the following expression

$P_{d}=1-0.819 e^{-14.63 \Lambda}-0.0976 e^{-89.22 \Lambda}-0.0325 e^{-228 \Lambda}-0.0509 e^{-125.9 \Omega^{2 / 3}}$

for the average deposition probability of a spherical particle in a cylindrical tube with Poiseuille flow. In the equation above the diffusion parameter $\Lambda$ is given by

$\Lambda=D_{p}{ }^{t} L / 4 U R^{2}$,

with $D_{p}{ }^{t}$ being the diffusion coefficient for a spherical particle. In order to apply Eq. (50) to non-spherical particles an appropriate diffusion coefficient must be used. At small rotational Peclet numbers random fiber orientation can be assumed, whereby the diffusion coefficient averaged over all directions is a good choice. The diffusional deposition efficiency at a tracheal flow rate of $375 \mathrm{~cm}^{3} / \mathrm{s}$ is shown for various generations of Weibel's lung model for fibers with equivalent mass diameter, $d_{e m}=0.01 \mu \mathrm{m}$, with $\beta=10$ and $\beta=100$, see Fig. 8 . The results for a spherical particle is included as well. In order to compare our results with those predicted by Ingham's formula, deposition is only considered over time $L / U$, which equals the mean residence time in the airway. As shown in the figure, the predictions are in good agreement for the case considered. 


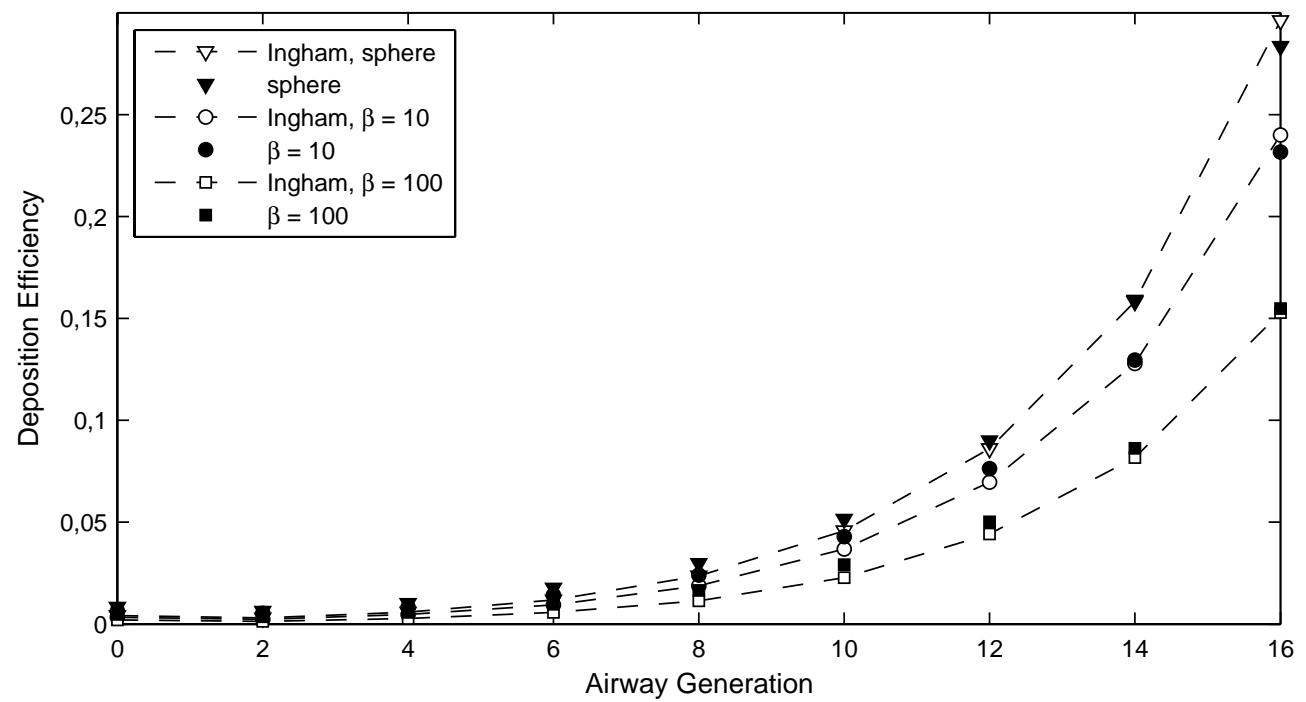

Figure 8. Deposition efficiency due to diffusion in a horizontal pipe with Poiseuille flow. Result for both a spherical particle and for fibers with varied aspect ratios are presented, and predictions using the formula of Ingham(1975) are included for comparison.

As a consequence of the fiber transport properties discussed earlier, deposition of both microfibers (with fixed $k$ ) and nanofibers decreases with increased fiber aspect ratio, up to a certain level, cf. Fig. 9. When fiber lengths approach the size of the airway diameter, however, deposition is rapidly increasing as a result of interceptive deposition. Results are presented for both a horizontal and an inclined airway for the microfibers. The reduction in deposition efficiency for the tube that is inclined 45 degrees is due to the reduced efficiency of gravitational settling, and for a vertical airway, the deposition due to sedimentation was less than $1 \%$. The deposition due to interception, naturally, is not a function of airway orientation, and deposition of nanofibers is indifferent to pipe direction as their motion is only marginally affected by gravity.

The influence of particle size and shape on the total deposition efficiency is shown in Fig. 10. In contrast to the results in Fig. 9, the deposition of microfibers is now increasing with fiber aspect ratio for all $\beta$, since particle mass is not held constant any longer. When visualizing the results in this form, the differences in deposition behavior between spherical and non-spherical particles of various diameters are obvious; for fibers with small aspect ratio, i.e. close to spherical shape, the region of minimum deposition is located around $0.5 \mu \mathrm{m}$, as expected from studies on spherical particles, while for particles with very elongated shape, the region of minimum deposition is shifted towards much smaller geometric diameters. 

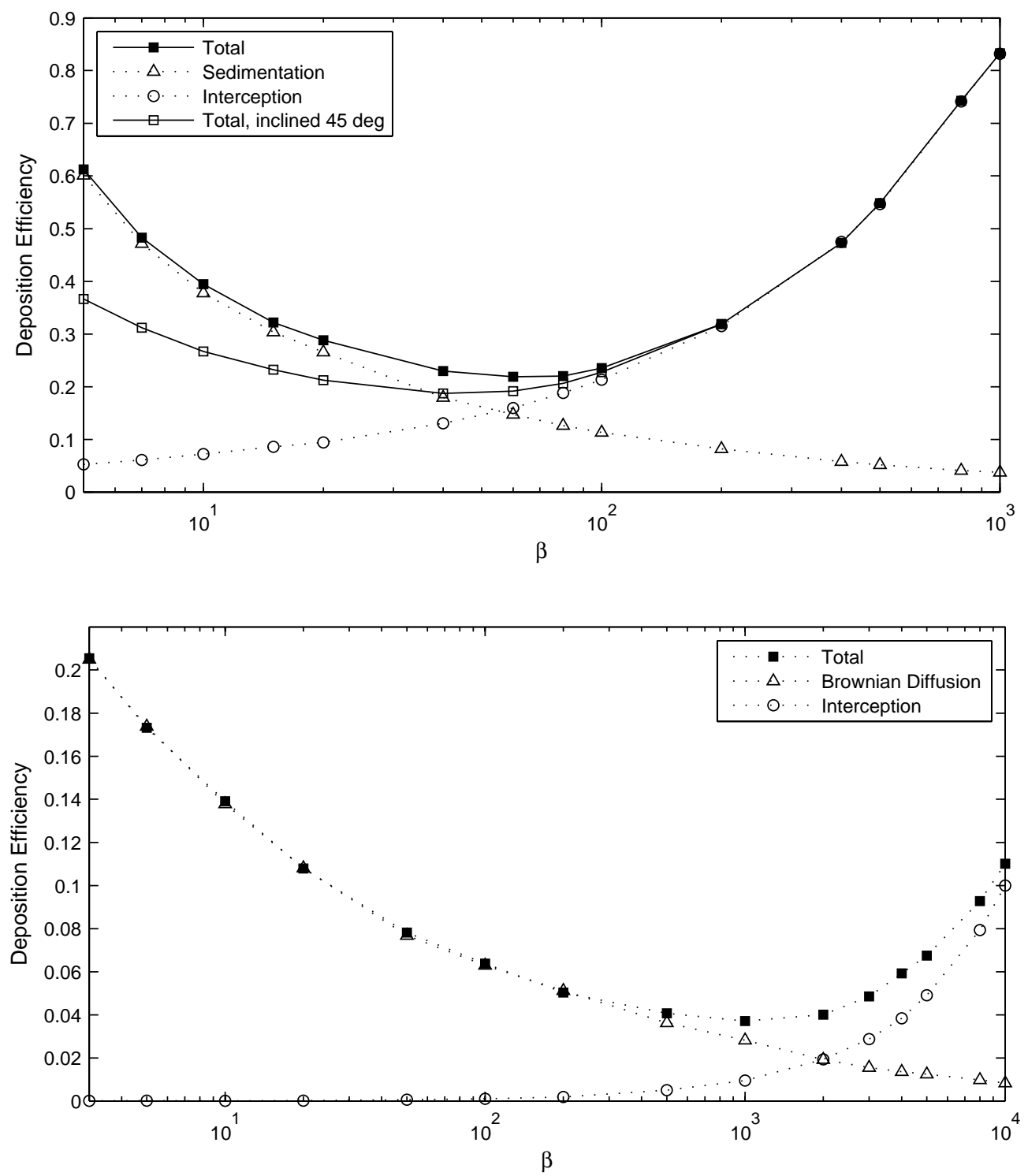

Fig 9. Size and shape dependence on deposition by sedimentation, Brownian diffusion and interception, both when acting separately and in combination. Results are shown for microfibers with $k=0.5$ in a horizontal and inclined airway (top), and nanofibers with $d_{f}=10 \mathrm{~nm}$ in a horizontal airway (bottom). 


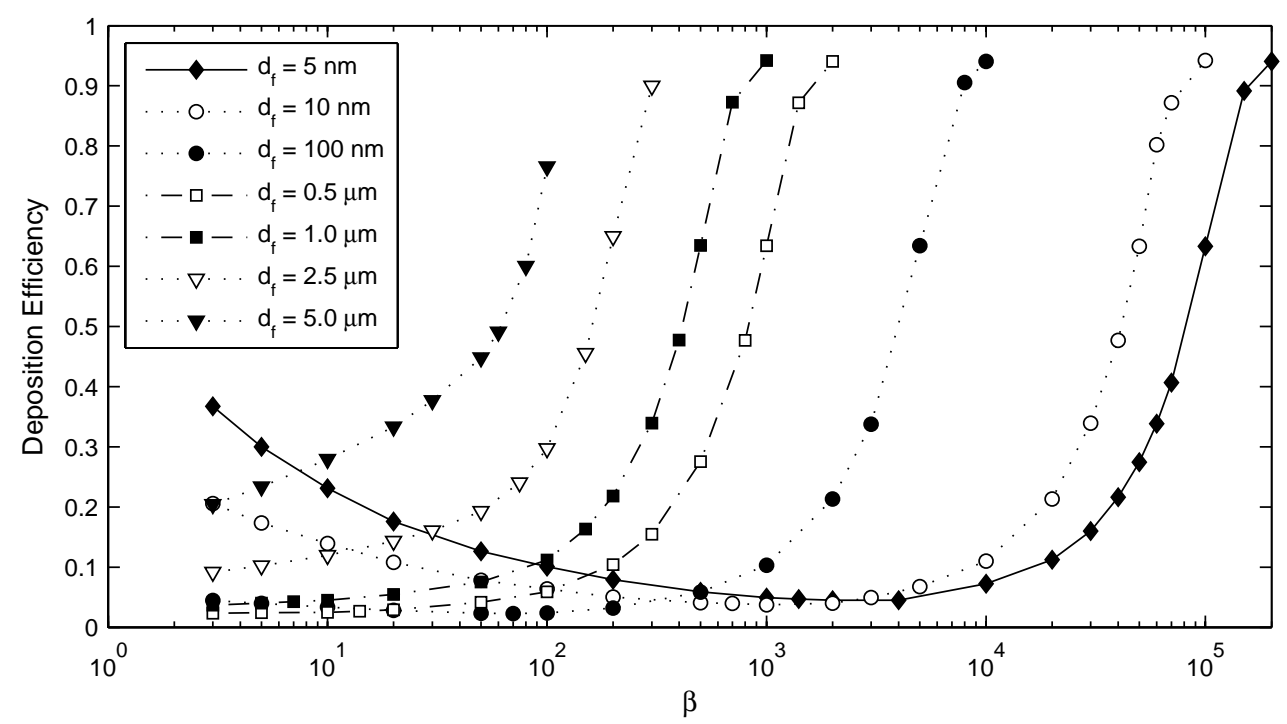

Figure 10. Dependence of fiber diameter and aspect ratio on fiber deposition.

To illustrate the effect of fiber length and to show differences in deposition behavior over different airway generations, deposition efficiency is plotted vs. fiber length for both G11 and G16 in Fig. 11. When fibers reach a certain length, interception is the only efficient deposition mechanism, whereby the deposition curves eventually coincide. Fibers of a few micrometers in length are least efficiently deposited, so that the majority of the fibers entering the terminal bronchiole may proceed to the deeper lung, where the more vulnerable alveoli are present. Fluid velocities and airway radii are generally larger in earlier generations of the respiratory airways, resulting in lower residence times and smaller fiber to airway ratios. Of this reason, fiber deposition is lower in G11 than in G16 for all deposition mechanisms considered in our model.

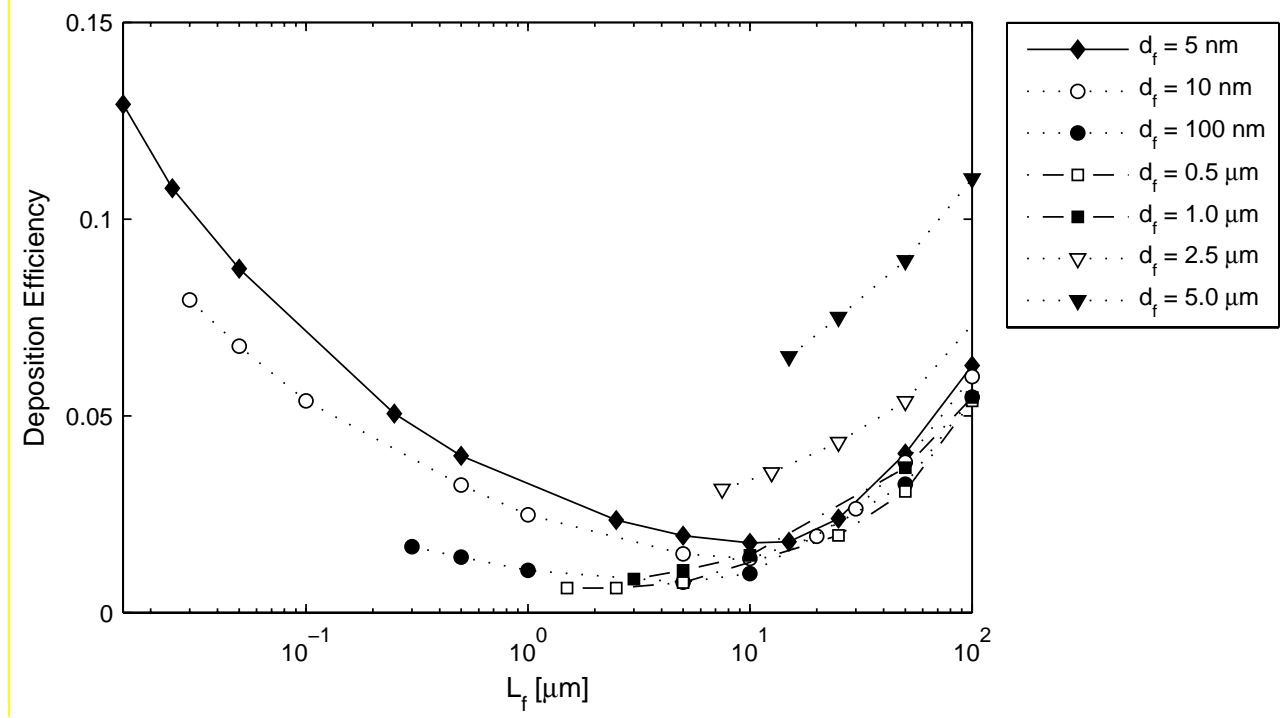




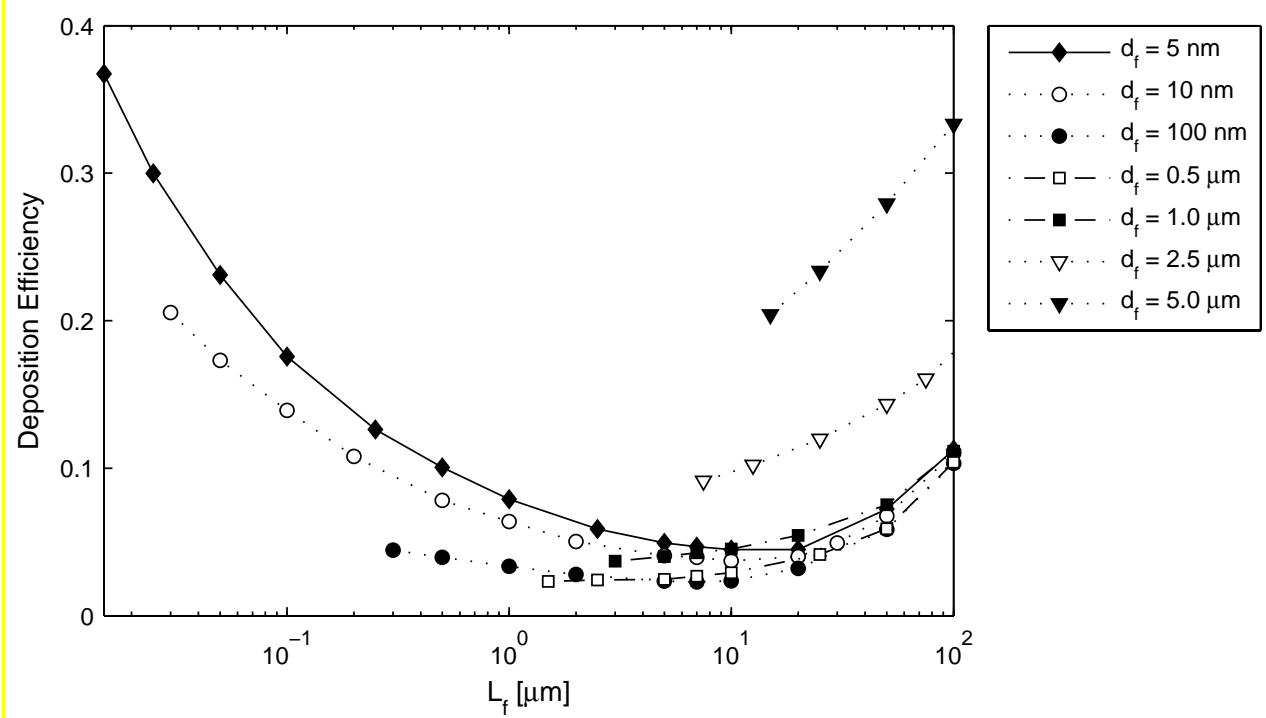

Figure 11. Dependence of fiber diameter and length on deposition efficiency in a horizontal pipe representing Weibel's G11 (top), and G16 (bottom). Note the difference in scale on the vertical axes.

As mentioned earlier, the probability of deposition of microfibers as well as nanofibers is most pronounced in smaller airways (as long as fiber inertia is negligible). However, there is a possibility of deposition in more proximal airways as well, and the deposition efficiency in G0 to G16 is shown in Fig. 12. The trends here are similar to those in Fig. 10, which showed the behavior in a single airway. Whereas fibers with small aspect ratios have minimum deposition for $1 \mu \mathrm{m}(0.5 \mu \mathrm{m}$ was not considered here but probably give lower deposition) over all single airway generations considered, the situation is quite different if regarding fibers with large aspect ratios, whereby the minimum deposition is received for fibers with diameters in the size-range $10-100 \mathrm{~nm}$, and lengths of several microns. Note that the multi-walled carbon nanotubes fall into this size-range, which is a reason for caution when handling these kind of materials. Microfibers are additionally deposited by inertial impaction, which adds to the deposition efficiency in the upper parts of the bronchial tree, this does not alter the regions of minimum deposition however, and deposition by impaction is believed to be comparatively low for the fibers and flow conditions presented here. 

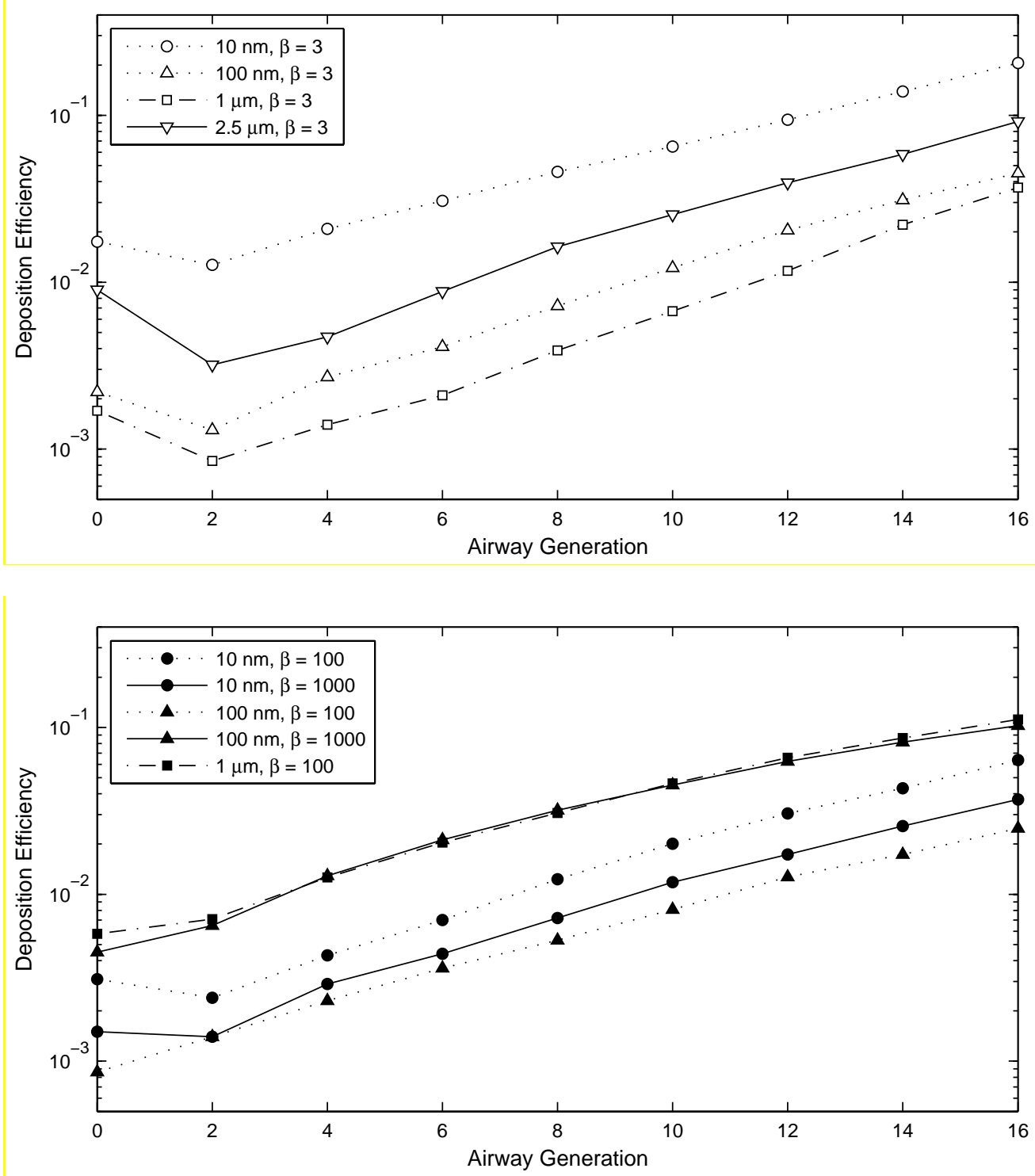

Figure 12. Deposition efficiency in various lung generations for fibers with diameters in the size-range $10 \mathrm{~nm}$ to 2.5 $\mu \mathrm{m}$, for $\beta=3$ (top) and $\beta=100 \& 1000$ (bottom) at light activity breathing.

Finally, to illustrate the influence of inhalation flow rate, the ratio of the deposition efficiency at light activity breathing to that of moderate activity breathing is shown in Fig. 13. The lower flow rate is close to half the higher one, which means that the mean residence is approximately twice as long at light activity breathing, and longer residence time means longer times for the fibers to settle or diffuse towards the airway walls. Recall from Eq. (42) that the size of the rms-Brownian fluctuations are proportional to $t^{1 / 2}$. From Fig. 13 we can see that for nanofibers with small aspect ratios, i.e., for fibers that are mainly affected by Brownian motion, the deposition ratio is indeed close to $\sqrt{2}$ in the first generations. In the smaller airways, however, the difference in deposition between the two 
flow rates are somewhat smaller. For fibers that are mainly deposited by gravitational settling the trend is similar, but with a linear dependence of time. Long fibers are mostly deposited by interception and then the dependence of the flow rate is less pronounced.

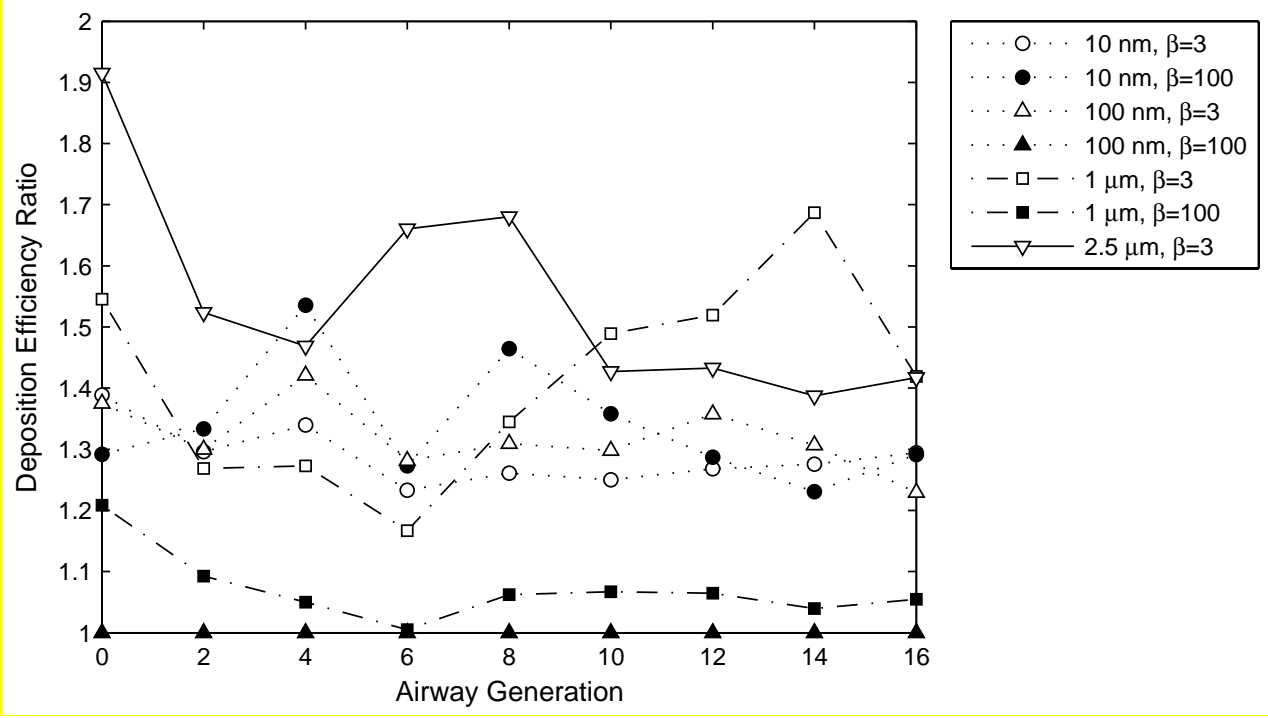

Figure 13. The ratio of the deposition efficiency at light activity breathing to that at moderate activity breathing for fibers of various sizes and shapes.

\section{Additional Discussion}

During inhalation at a steady rate, the velocity in the airways increases slightly until the air reaches the lobar bronchi. After that, the velocity decreases rapidly as a result of the tremendous increase in the total airway cross-sectional area that follows from the large number of small airways. In the trachea and main bronchi, the airflow can be turbulent at peak inspiratory and expiratory flow rates for the normal breathing cycle, but the remaining airflow is laminar under normal conditions (Hinds, 1999). Because the airway sections are relatively short compared with their diameters, the airflow in the respiratory airways is not fully developed in general. This further complicates mathematical analysis and modeling. In the deep lung, however, we have low airway Reynolds numbers, $R e=$ $2 R U / v$, whereby the flow profile develops rather fast. In G16 we have $R e=2$, for example, whereby the flow is fully developed within one pipe diameter, and then the assumption of fully developed flow is a reasonable approximation. During actual breathing, the air flow rate is continuously changing and reverses direction twice each cycle (Hinds, 1999). The application of the model on other flow fields, including transient effects, is left for future work. 
Finally we will discuss some of the assumptions introduced. To start with, fibers are assumed straight and rigid. In reality, long CNTs are flexible in the direction perpendicular to their axis; experimental work, however, suggests that nanotubes tend to align during shear, and theoretical work has also showed that nanotubes, like macro fibers, become straight and aligned along the shearing direction as time proceeds (e.g. Tang and Advani, (2008)). Inertial terms are neglected in both the equation for fiber translation and rotation. The effect of inertia on the translational motion is omitted due to the small Stokes numbers considered, and neglecting the inertia on fiber rotation is consistent with the results of Asgharian and Anjilvel (1995b) who showed that inertial effects on fiber rotation in a shear flow only have to be included if considering fibers with diameters above $1 \mu \mathrm{m}$ in the conducting airways, and we are primarily interested in flow in deeper parts of the lungs.

\section{CONCLUSIONS}

A semi-analytical model describing the motion of fibrous particles ranging from nano- to micro scale was developed, and some important differences between respiratory transport and deposition properties of fibrous particles of various sizes and shapes were elucidated. The focus of this work was on fiber motion in the respiratory airways, although the model is valid for general flows and geometries at small particle Reynolds and Stokes numbers.

The fiber equations of motion in their final form enable us to obtain valuable information on fiber behavior, since from these it is apparent which parameters influence fiber motion, and to what extent. It is well known that decrease in particle size means reduced efficiency of sedimentation but increased intensity of Brownian diffusion, as confirmed by our model. Additionally, if regarding the effects due to particle shape alone (by varying the aspect ratios and diameters of the microfibers simultaneously such that the effect of particle mass does not come into play), our model suggests that deposition both due to gravitational settling and Brownian diffusion decreases with increased fiber aspect ratio. Comparisons between our model and simpler models on the prediction of deposition by sedimentation (Pich, 1972) or diffusion (Ingham, 1975) alone show good agreement. Besides validating our model this also authorize the usage of these simpler models to predict deposition by a single mechanism. Notice that this conclusion only holds for the cases studied and further studies are required before general conclusions can be made.

If considering the combined effect of fiber size and shape, there exist various combinations of these giving minimum deposition efficiency. For fibers with small aspect ratios, our results suggest that the minimum deposition occurs for fiber diameters around $0.5 \mu \mathrm{m}$, as expected from the work on spherical particles, while for fibers with 
larger aspect ratios, the minimum is shifted towards smaller diameters. If considering the combined effect of fiber size and shape, our results suggest that for particles with elongated shape, those with diameters in the size-range range 10-100 $\mathrm{nm}$ and lengths of several micrometers have the highest possibility to reach the vulnerable gasexchange region in the deep lung. Note that the popular multi-walled carbon nanotubes fall into this size-range.

\section{ACKNOWLEDGEMENTS}

This work was made possible by funding from the Swedish Research Council, the Kempe foundation, and the Sweden-America foundation. The authors also acknowledge fruitful discussions with Professor Thomas Sandström at the Department of Public Health and Clinical Medicine at Umeå University.

\section{REFERENCES}

Asgharian, B., and Anjilvel, S. (1995a). Movement and Deposition of Fibers in an Airway with Steady ViscousFlow. Aerosol Sci. Technol., 22(3): 261-270.

Asgharian, B., and Anjilvel, S. (1995b). The Effect of Fiber Inertia on Its Orientation in a Shear Flow with Application to Lung Dosimetry. Aerosol Sci. Technol., 23(3): 282-290.

Chen, Y. K., and Yu, C. P. (1991). Sedimentation of Fibers from Laminar Flows in a Horizontal Circular Duct. Aerosol Sci. Technol., 14(3): 343 - 347.

Dahneke, B. E. (1973a). Slip Correction Factors for Nonspherical Bodies: II. Free Molecule Flow. J. Aerosol Sci., 4(2): 147-161.

Dahneke, B. E. (1973b). Slip Correction Factors for Nonspherical Bodies: III. The Form of the General Law. J. Aerosol Sci., 4(2): 163-170.

Donaldson, K., Aitken, R., Tran, L., Stone, V., Duffin, R., Forrest, G., and Alexander, A. (2006). Carbon Nanotubes: A Review of Their Properties in Relation to Pulmonary Toxicology and Workplace Safety. Toxicol. Sci., 92(1): 5-22.

Fan, F.-G., and Ahmadi, G. (2000). Wall Deposition of Small Ellipsoids from Turbulent Air Flows: A Brownian Dynamics Simulation. J. Aerosol Sci., 31(10): 1205-1229.

Fan, Z., and Advani, S. G. (2005). Characterization of Orientation State of Carbon Nanotubes in Shear Flow. Polymer, 46(14): 5232-5240.

Gogotsi, Y. (2003). How Safe Are Nanotubes and Other Nanofilaments? Mater. Res. Innovations, 7(4): 192-194. 
Goldstein, H. (1980). Classical Mechanics. Addison-Wesley, Reading, MA.

Higham, D. J. (2001). An Algorithmic Introduction to Numerical Simulation of Stochastic Differential Equations. SIAM Rev., 43(3): 525-546.

Hinds, W. C. (1999). Aerosol Technology: Properties, Behavior, and Measurement of Airborne Particles. Wiley, New York.

ICRP Task Group on Lung Dynamics (1966). Deposition and Retention Models for Internal Dosimetry of the Human Respiratory Tract. Health. Phys., 12: 173-207.

Ingham, D. B. (1975). Diffusion of Aerosols from a Stream Flowing through a Cylindrical Tube. J. Aerosol Sci., 6(2): $125-132$.

Jeffery, G. B. (1922). The Motion of Ellipsoidal Particles Immersed in a Viscous Fluid. Proc. R. Soc. London., 102(175): 161-179.

Johnson, D. L. (1994). Behavior of Inhaled Fibers - Potential Applications to Medicinal Aerosols. Part. Sci. Technol., 12(2): 161-173.

Kleinstreuer, C., and Zhang, Z. (2010). Airflow and Particle Transport in the Human Respiratory System. Annu. Rev. Fluid Mech., 42(1): 301-334.

Kloeden, P. E., and Platen, E. (1999). Numerical Solution of Stochastic Differential Equations. Springer-Verlag, Berlin.

Oberdörster, G., Maynard, A., Donaldson, K., Castranova, V., Fitzpatrick, J., Ausman, K., Carter, J., Karn, B., Kreyling, W., Lai, D., Olin, S., Monteiro-Riviere, N., Warheit, D., and Yang, H. (2005). Principles for Characterizing the Potential Human Health Effects from Exposure to Nanomaterials: Elements of a Screening Strategy. Part. Fibre Toxicol., 2(1): 8.

Phalen, R. F., and Hoover, M. D. (2006). Aerosol Dosimetry Research Needs. Inhal. Toxicol., 18(10): 841 - 843.

Pich, J. (1972). Theory of Gravitational Deposition of Particles from Laminar Flows in Channels. J. Aerosol Sci., 3(5): $351-361$.

Poland, C. A., Duffin, R., Kinloch, I., Maynard, A., Wallace, W. A. H., Seaton, A., Stone, V., Brown, S., MacNee, W., and Donaldson, K. (2008). Carbon Nanotubes Introduced into the Abdominal Cavity of Mice Show Asbestos-Like Pathogenicity in a Pilot Study. Nat. Nanotechnol., 3(7): 423-428.

Rapaport, D. C. (1997). The Art of Molecular Dynamics Simulation. Cambridge university press, Cambridge. 
Sturm, R., and Hofmann, W. (2006). A Computer Program for the Simulation of Fiber Deposition in the Human Respiratory Tract. Computers in biology and medicine, 36: 1252-1267.

Swaminathan, T. N., Mukundakrishnan, K., and Hu, H. H. (2006). Sedimentation of an Ellipsoid inside an Infinitely Long Tube at Low and Intermediate Reynolds Numbers. J. Fluid Mech., 551(-1): 357-385.

Tang, W., and Advani, D. (2008). Dynamic Simulation of Carbon Nanotubes in Simple Shear Flow. CMES, 25(3): $149-164$.

Tucker, C. L., and Advani, S. G., (1994). Processing of Short-Fiber Systems, in Composite Materials Series: Flow and Rheology in Polymer Composites Manufacturing. R. B. Pipes, ed., Elsevier, Amsterdam.

Weibel, E. R. (1963). Morphometry of the Human Lung. Academic Press, New York.

Yeh, H. C., Schum, G. M., and Duggan, M. T. (1979). Anatomic Models of the Tracheobronchial and Pulmonary Regions of the Rat. The Anatomical Record, 195(3): 483-492.

Zhang, H., Ahmadi, G., Fan, F. G., and McLaughlin, J. B. (2001). Ellipsoidal Particles Transport and Deposition in Turbulent Channel Flows. Int. J. Multiphase Flow, 27: 971-1009.

Åkerstedt, H. O., Lundström, T. S., and Högberg, S. M. (2007). Electrostatic Filtration of Air-Borne Nano-Particles. J. Nanostruct. Polym. Nanocompos., 3(4): 111-115.

\section{APPENDIX A}

\section{Derivation of the slip correction factors for fibers}

The main idea of this method is to calculate the size of an adjusted sphere with the same slip correction factor as the non-spherical body in free molecule flow. The adjusted sphere also has the same correction factor in the continuum regime, since its value is unity in this case. Thus, the slip correction factors of the adjusted sphere are valid over the whole range of Knudsen numbers, $K n=2 \lambda / d_{f}$. The translational slip correction factors for the ellipsoid can be expressed in the form of Cunningham's correction factor as

$C_{i^{\prime}}^{t}=1+\frac{d_{f}}{2 r_{i^{\prime}}} K n\left[1.257+0.4 \exp \left(\frac{-2.2 r_{i^{\prime}}}{d_{f} K n}\right)\right]$

The adjusted radii are defined as 


$$
\begin{aligned}
r_{\|}{ }^{t} & =\frac{1.657 d_{f} \beta}{16\left(\beta^{2}-1\right)}\left[\frac{2 \beta^{2}-1}{\sqrt{\beta^{2}-1}} \ln \left(\beta+\sqrt{\beta^{2}-1}\right)-\beta\right] \\
& \times\left[2 \Lambda_{1} f+\frac{\Lambda_{3}}{\Lambda_{2}{ }^{2}}\left\{\Lambda_{2}^{2}(4-2 f)-4+\left(3-\frac{\pi}{2 \beta^{2}}\right) f\right\}\right] \\
r_{\perp}{ }^{t} & =\frac{1.657 d_{f} \beta}{32\left(\beta^{2}-1\right)}\left[\frac{2 \beta^{2}-3}{\sqrt{\beta^{2}-1}} \ln \left(\beta+\sqrt{\beta^{2}-1}\right)+\beta\right] \\
& \times\left[\Lambda_{1}\left\{4+\left(\frac{\pi}{2}-1\right) f\right\}+\frac{\Lambda_{3}}{\Lambda_{2}^{2}}\left\{2+\frac{4 \Lambda_{2}^{2}+\pi-6}{4} f\right\}\right]
\end{aligned}
$$

for translational motion parallel and perpendicular to the flow direction, respectively. The momentum accommodation coefficient, $f$, is set to 0.9 , following recommendations in Dahneke (1973a), and

$\Lambda_{1}=\frac{\sin ^{-1}\left(\Lambda_{2}\right)}{\Lambda_{2}}, \Lambda_{2}=\sqrt{1-\frac{1}{\beta^{2}}}, \Lambda_{3}=\frac{1}{\beta}-\Lambda_{1}$.

The adjusted radius for a cylinder rotating about its minor axis is given by

$$
\begin{aligned}
r_{\perp}{ }^{r} & =\frac{4.971 d_{f}}{64}[\ln (2 \beta)+0.1931] \\
& \times\left[1+\frac{1}{\beta}+\frac{1}{\beta^{2}}+\frac{1}{2 \beta^{3}}+f\left(\frac{\pi-6}{12}-\frac{1}{2 \beta}-\frac{1}{\beta^{2}}+\frac{\pi-4}{16 \beta^{3}}\right)\right],
\end{aligned}
$$

and substitution in Eq. (A1) gives the rotational slip correction factor. 